\title{
Dielectric studies on Sm-modified two-layered BLSF ceramics
}

\author{
MD ABDUL BASHEER, G PRASAD, G S KUMAR and N V PRASAD* \\ Material Research Laboratory, Department of Physics, Osmania University, Hyderabad 500007, India \\ *Author for correspondence (nvp1969@ rediffmail.com, nvp_phy@osmania.ac.in)
}

MS received 20 May 2018; accepted 27 September 2018; published online 23 April 2019

\begin{abstract}
Sm}_{x} \mathrm{Bi}_{3-x} \mathrm{TiNbO}_{9}(\mathrm{SBTN})$ with $x=0,0.2,0.4,0.6,0.8$ and 1.0, a novel type of ceramic was prepared by a conventional solid-state reaction method. X-ray diffraction patterns confirm the formation of a single-phase, and lattice parameters were calculated based on the parent compound $\mathrm{Bi}_{3} \mathrm{TiNbO}_{9}(\mathrm{BTN})$. Well-defined grains were seen in the scanning electron microscopy pictures. A kink observed near $400^{\circ} \mathrm{C}$ for all the samples in the dielectric plot as well as in the stretched exponential parameter $(\beta) v s$. temperature plot reveals the dielectric relaxation of the samples. The tolerance factor $(t)$ was found to increase with increase in the size of the A-site (Bi-site) modified ion ( $\mathrm{Sm}$ ). Impedance and modulus spectroscopic studies were conducted to understand the plausible reason for the dielectric relaxation. The relaxation was found to be well-fit with Arrhenius equation. SBTN samples showed lower dielectric loss compared to BTN. The remnant polarization, obtained from experimental, was found to be less than that of theoretically calculated polarization, using Shimakawa's formula. The proposed defect mechanism was interpreted using FTIR and Raman spectroscopic studies.
\end{abstract}

Keywords. Bismuth-layered structure ferroelectric (BLSF); dielectric relaxation; spontaneous polarization; defect mechanism; stretched exponential parameter $(\beta)$.

\section{Introduction}

The Aurivillius family of bismuth-based layered structure ferroelectric (BLSF) perovskite materials has potential applications in high temperature piezoelectric and ferroelectric random access memory (FeRAM) devices [1-3]. BLSF is a common acronym for Aurivillius ferroelectric materials [4-7]. The general formula of the BLSF materials is described by: $\left(\mathrm{Bi}_{2} \mathrm{O}_{2}\right)^{2+}\left(\mathrm{A}_{n-1} \mathrm{~B}_{n} \mathrm{O}_{3 n+1}\right)^{2-}$, where perovskite blocks $\left(\mathrm{A}_{n-1} \mathrm{~B}_{n} \mathrm{O}_{3 n+1}\right)^{2}$ are sandwiched between the bismuth oxide layers. Here, A-site can be readily occupied by mono-, di-, tri-valent and rare-earth elements (in the present case, it is $\mathrm{Bi} / \mathrm{Sm})$. Similarly, B-site can be decorated with tetra-, penta- or hexa-valent ions (in the present case, it is $\mathrm{Nb} / \mathrm{Ti}$ ). The notation ' $n$ ' represents the number of octahedral layers in the layered-perovskite slab (in the present case $n=2$ ). Newnham et al [8] reported that $\mathrm{Sr}^{2+}$ and $\mathrm{Ba}^{2+}$ substituted layered compounds showed lower Curie temperature, compared to the parent compound $\left(\mathrm{Bi}_{3} \mathrm{TiNbO}_{9}\right)$. Later, many researchers demonstrated that $\mathrm{Bi}$-site modified $\mathrm{Bi}_{3} \mathrm{TiNbO}_{9}$ is a good ferroelectric with the highest Curie temperature $\left(940^{\circ} \mathrm{C}\right)$ among the other materials reported in the BLSF family [9-13]. One of the factors for observing such phenomenon was attributed to reduced-distortion in the perovskite units [9-17]. Among BLSF systems, strontium bismuth-tantalate $\left(\mathrm{SrBi}_{2} \mathrm{Ta}_{2} \mathrm{O}_{9}\right)$ and strontium bismuth-niobate $\left(\mathrm{SrBi}_{2} \mathrm{Nb}_{3} \mathrm{O}_{9}\right)$ are found to be the most promising candidates for non-volatile FeRAM devices $[9,12]$. A noteworthy aspect from the literature is that the spontaneous polarization $\left(P_{\mathrm{s}}\right)$ lies along the $c$-axis. The value of polarization $\left(P_{\mathrm{s}}\right)$ varies depending on whether there is an even or odd number of layers $(n)$ in the BLSF compound [13]. Earlier studies predicted that donor and acceptor doping would affect the ferroelectric properties. These predictions were well reflected in $P$ vs. $E$ loops, observed by Yan $e t$ al [9]. They found the dielectric loss peaks near $200^{\circ}$ for acceptor doped BTNO $\left(\mathrm{Bi}_{3} \mathrm{Nb}_{0.8} \mathrm{Ti}_{1.2} \mathrm{O}_{9}\right)$ materials. The relaxation peak was attributed to oxygen-ion jump mechanism [9]. The dielectric constant was found to be higher for acceptor doped BTNO $\left(\mathrm{Bi}_{3} \mathrm{Nb}_{0.8} \mathrm{Ti}_{1.2} \mathrm{O}_{9}\right)$, compared to donor doped BTNO $\left(\mathrm{Bi}_{3} \mathrm{Nb}_{1.2} \mathrm{Ti}_{0.8} \mathrm{O}_{9}\right)$. Sugaya et al [15] reported dielectric properties on $\mathrm{RBi}_{2} \mathrm{TiNbO}_{9}(\mathrm{R}=\mathrm{La}, \mathrm{Sm}, \mathrm{Nd}, \mathrm{Gd})$ ceramics. In their investigation, $\mathrm{Sm}_{x} \mathrm{Bi}_{2} \mathrm{TiNbO}_{9}$ (where $x=0-1$ ) compound of all the compositions showed a higher transition temperature in the range of $800-950^{\circ} \mathrm{C}$. It has been reported that the Curie temperature can be decreased by decreasing the size of the Asite cation modification for the Bi-site [5]. Our recent report [14] on La-modified BTN $\left(\mathrm{Bi}_{2.45} \mathrm{La}_{0.55} \mathrm{TiNbO}_{9}\right)$ and similar compounds showed a relaxor-kind behaviour. The dielectric peaks were found to be broad and well-fit to the modified Curie-Weiss law and Vogel-Fulcher relation. The enhancement of dielectric constant can be attributed to the Bi-vacancy, produced by La-doping at the Bi-site (A-site) in the perovskite cell, which makes the domain wall movement more easier. In addition, the mechanical quality factors $\left(Q_{\mathrm{r}}\right)$ of these samples were found to be comparable to that of $\mathrm{Pb}$-based materials [13]. Keeping in view of all aforementioned, we have prepared $\mathrm{Bi}_{3-x} \mathrm{Sm}_{x} \mathrm{TiNbO}_{9}(x=0.2,0.4,0.6,0.8,1.0)$ ceramics by a solid-state reaction method. The dielectric and impedance 
properties of the samples were studied. Raman spectroscopic studies were also conducted.

\section{Experimental}

$\mathrm{Bi}_{3-x} \mathrm{Sm}_{x} \mathrm{TiNbO}_{9}(x=0.2,0.4,0.6,0.8,1.0)$ ceramics were prepared by the traditional solid-state reaction method. The reactant oxide powders were AR grade with $99.99 \%$ purity. The powders of $\mathrm{Bi}_{2} \mathrm{O}_{3}, \mathrm{Sm}_{2} \mathrm{O}_{3}, \mathrm{TiO}_{2}$ and $\mathrm{Nb}_{2} \mathrm{O}_{5}$ were taken in a stoichiometric ratio. The powders were mixed thoroughly using agate mortar and pestle. The dried mixture was heated (pre-sintered) at $900^{\circ} \mathrm{C}$ for $2 \mathrm{~h}$. Requisite amount of polyvinyl alcohol was added as a binder before making it into pellets. Circular-shaped pellets were made by applying uni-axial stress of 2 tonnes $\mathrm{cm}^{-2}$ (the pressure is roughly about $200 \mathrm{MPa}$ ). The pressed pellets were once again heated (final-sintered) at $1050-1100^{\circ} \mathrm{C}$ for $5 \mathrm{~h}$. To measure the impedance and dielectric measurements, ohmic contacts were made on the both sides of the surface of the pellets. Impedance and dielectric measurements were made by Hewlett-Packard (HP4192A) impedance analyser. The X-ray diffraction (XRD) patterns were obtained by using a Philips X-Pert diffractometer with $\mathrm{CuK} \alpha$ radiation. The diffractometer was operated at $40 \mathrm{kV}$ and $30 \mathrm{~mA}$. The $2 \theta$ range is made between 10 and $90^{\circ}$ with the scan step of $0.02^{\circ}$.

\section{Results and discussion}

XRD patterns obtained at room temperature is shown in figure 1. A maximum intensity (characteristic) peak of 115 is observed for all the samples. No secondary phase is observed in the XRD pattern. This clearly confirms the formation of a single phase. The intensity of $\left(\begin{array}{lll}0 & 0 & 2\end{array}\right)$ is observed near $33^{\circ}$ of $2 \theta$ value corresponding to even reflections of the samples. This is considered to be the characteristic nature of the BLSF samples, where the possible preferred orientation is possible along $\left(\begin{array}{lll}0 & 0 & l\end{array}\right)$ reflections. All the XRD peaks were indexed according to standard powder diffraction data of the parent compound. The lattice parameters of $\mathrm{Sm}_{x} \mathrm{Bi}_{3-x} \mathrm{TiNbO}_{9}$ (SBTN) samples are calculated based on the parent compound $\mathrm{Bi}_{3} \mathrm{TiNbO}_{9}$. The lattice parameters, interlayer distance $(c / n)$, distortion, theoretical and experimental density values are summarized in table 1 . The scanning electron microscopy (SEM) photographs are depicted in figure 2. All the grains are found to have a plate-like structure, which is being considered as typical morphology of BLSF materials. The average size of the grain was found to be $\sim 4 \mu \mathrm{m}$. The SEM images show a compact form and no pores were seen in between the grains.

BTNO has $\mathrm{A} 2{ }_{i}$ am orthorhombic symmetry and polarization along the $a$-axis. The spontaneous polarization in BTNO is caused by different atomic displacements in the composition namely $\mathrm{Bi}, \mathrm{Nb} / \mathrm{Ti}$ and $\mathrm{O}$ ions from their respective positions along the $a$-axis. The polarization caused by $b$ - and

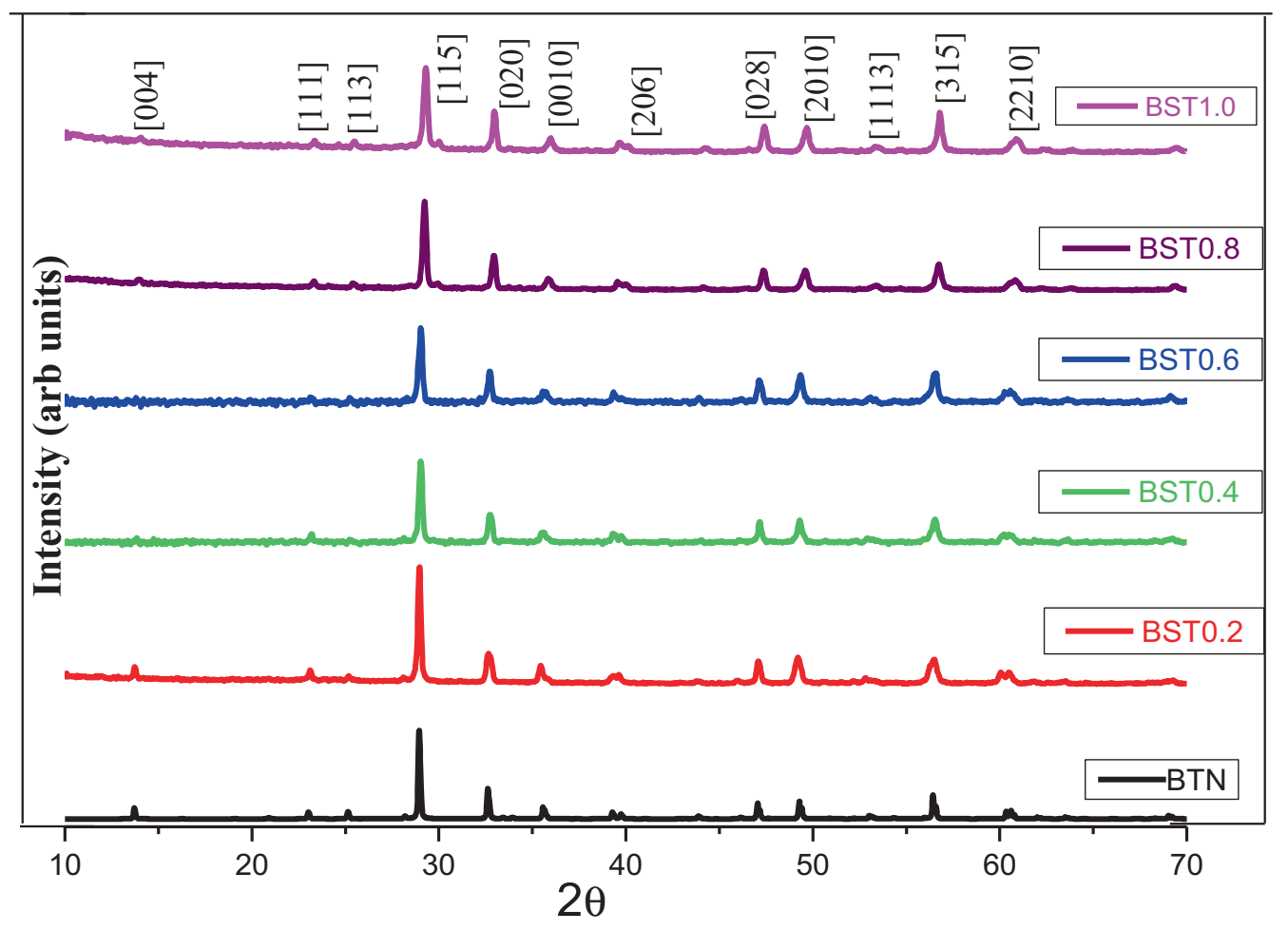

Figure 1. XRD pattern of BTN (parent compound) and SBTN- $x$. 
Table 1. Lattice parameters of BTN and SBTN- $x(x=0.2,0.4,0.6,0.8$ and 1.0).

\begin{tabular}{|c|c|c|c|c|c|c|c|}
\hline Compound & $\begin{array}{c}\text { Lattice } \\
\text { parameters }(\AA)\end{array}$ & Volume $(V)\left(\AA^{3}\right)$ & $\begin{array}{l}\text { Orthorhombic } \\
\text { distortion }(b / a)\end{array}$ & $c / a$ & $\begin{array}{c}\text { Inter layer } \\
\text { distance }(c / n)\end{array}$ & $\begin{array}{c}\text { Experimental } \\
\text { density }\left(\mathrm{g} \mathrm{cm}^{-3}\right)\end{array}$ & $\begin{array}{c}\text { Theoretical } \\
\text { density }\left(\mathrm{g} \mathrm{cm}^{-3}\right)\end{array}$ \\
\hline BTN & $\begin{array}{l}a=5.400 \\
b=5.402 \\
c=24.86\end{array}$ & 725.20 & 1.000 & 4.603 & 12.43 & - & - \\
\hline BST- 0.2 & $\begin{array}{l}a=5.427 \\
b=5.465 \\
c=25.59\end{array}$ & 759.26 & 1.007 & 4.71 & 12.79 & 7.74 & 7.87 \\
\hline BST-0.4 & $\begin{array}{l}a=5.420 \\
b=5.456 \\
c=25.50\end{array}$ & 754.34 & 1.006 & 4.704 & 12.75 & 7.54 & 7.82 \\
\hline BST-0.6 & $\begin{array}{l}a=5.371 \\
b=5.434 \\
c=25.57\end{array}$ & 749.59 & 1.011 & 4.760 & 12.78 & 7.58 & 7.77 \\
\hline BST-0.8 & $\begin{array}{l}a=5.356 \\
b=5.385 \\
c=25.16\end{array}$ & 721.74 & 1.005 & 4.696 & 12.58 & 7.68 & 7.96 \\
\hline BST-1.0 & $\begin{array}{c}a=5.390 \\
b=5.426 \\
c=25.046\end{array}$ & 732.56 & 1.006 & 4.646 & 12.52 & 7.46 & 7.73 \\
\hline
\end{tabular}

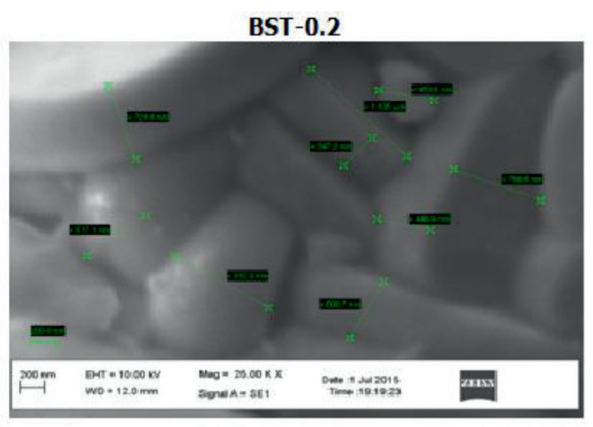

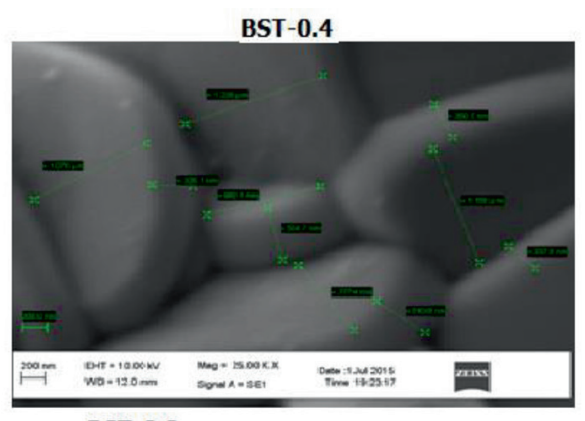

BST-0.8
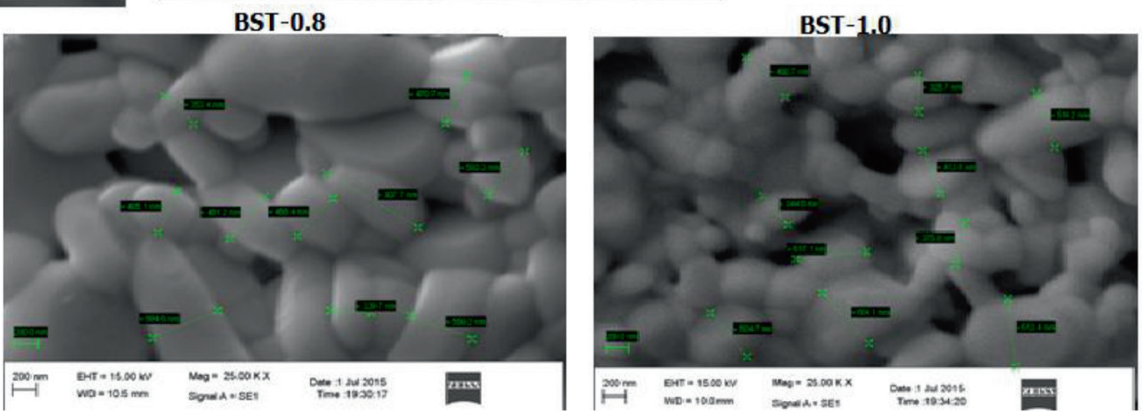

Figure 2. SEM micrographs of SBTN-x.

$c$-axes gets cancelled and the total polarization $(P)$ is only due to the displacement of constituent ion in the $a$-direction. The $P$ can be calculated by using the following Shimakawa formula:

$$
P=\sum \frac{\left(\Delta x_{\mathrm{i}} Q_{\mathrm{ie}}\right)}{V}
$$

where $\Delta x_{\mathrm{i}}$ represents the multiply of an atomic displacement of the constituent ion in the $a$-axis. $Q_{\text {ie }}$ and $V$ are the ionic charge and volume of the unit cell, respectively. The polarization $v s$. electric field ( $P v s . E)$ loops obtained at room temperature is depicted in figure $3 \mathrm{a}$. The variation of remnant polarization $\left(P_{\mathrm{r}}\right)$, obtained from the hysteresis loops, with the Sm-composition for all the present samples 

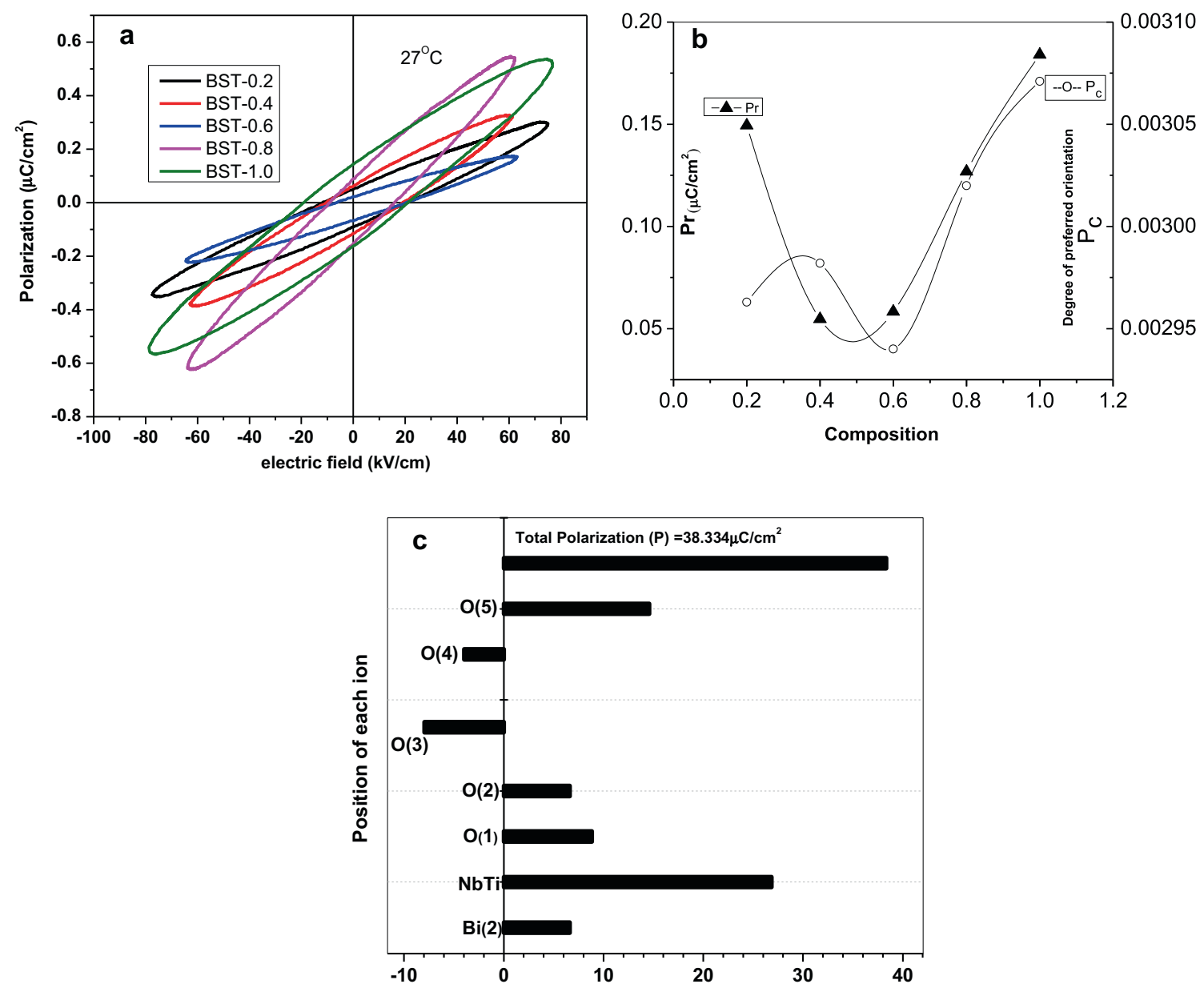

Figure 3. (a) Polarization $v s$. electric field ( $P-E$ loop) of SBTN- $x$ at room temperature. (b) Variation of remnant polarization $\left(P_{\mathrm{r}}\right)$ and degree of preferred orientation $\left(P_{\mathrm{c}}\right)$ with the Sm composition $(\mathrm{SBTN}-x)$. (c) Total polarization $(P)$ of the parent compound BTN with different ionic positions (theoretically calculated).

is shown in figure $3 \mathrm{~b}$. The degree of preferred orientation $\left(P_{\mathrm{c}}\right)$ of the $c$-axis was calculated by using the formula $P_{\mathrm{c}}=$ $\sum I(00 l) / \sum I(h k l)$, where $\sum I(00 l)$ summation of all the intensities of X-ray reflection occurs in $(00 l)$ planes and $\sum I(h k l)$ is the summation of all the $(h k l)$ planes. The variation of $P_{\mathrm{c}}$ with the $\mathrm{Sm}$ composition is also shown in figure $3 \mathrm{~b}$. The total polarization of BTNO due to the different constituent ions, calculated by equation (1), is shown in figure 3c. From the plot (figure 3b), it is evident that the remnant polarization increases with increasing $\mathrm{Sm}$ composition and $P_{\mathrm{c}}$ decreases with the composition up to 0.6 and thereafter, it increases with the $\mathrm{Sm}$ composition. The values of remnant polarization and coercive field were found to be low, indicating the typical nature of layered-ferroelectric materials. Observation of non-saturated hysteresis loops and the typical ferroelectric behaviour is generally attributed to the higher ionic conductivity and the presence of complex-defect dipoles. The higher conductivity of the samples arises due to the movement of oxygen vacancies which generally arises during the sintering process. The complex dipoles require high electric fields to switch the polarization process, and hence, the samples should have high dielectric strength, so that the sample can withstand even at a higher applied electric field. Similar results were observed in five layer compound [13].

The characteristic of ionic displacement is dependent on structural factors which include ionic size, tolerance factor and ionic polarizability. Here, it should be noted that the changes in $\mathrm{A}-\mathrm{O}$ and $\mathrm{B}-\mathrm{O}$ bond lengths would result in the octahedral tilt at A-site and B-site and hence, in ferroelectric properties. The tolerance factor $(t)$ of samples prepared in the present investigation with various compositions ( $\mathrm{Sm}$ ) was calculated by using the following formula:

$$
t=\frac{R_{\mathrm{O}}+(3-x) R_{\mathrm{Bi}}+x R_{\mathrm{Sm}}}{\sqrt{2}\left[R_{\mathrm{O}}+\left(R_{\mathrm{Ti}}+R_{\mathrm{Nb}}\right)\right]}
$$

$R_{\mathrm{O}}$ is the oxygen ionic radius, $R_{\mathrm{Bi}}$ and $R_{\mathrm{Sm}}$ are the A-site occupied ionic radii and $R_{\mathrm{Ti}}$ and $R_{\mathrm{Nb}}$ are the $\mathrm{B}$-site ionic radii, respectively. If the tolerance factor is 1 , then, it indicates the perfect fit. For most of the distorted perovskite materials, $t$ is above $0.8(0.8<t<1)$. 
The variation of the tolerance factor with the $\mathrm{Sm}$ composition is shown in figure 4. Here, it should be noted that the changes in $\mathrm{A}-\mathrm{O}$ and $\mathrm{B}-\mathrm{O}$ bond lengths would result in the octahedral tilt at A-site and $\mathrm{B}$-site and hence, in ferroelectric properties. The result is consistent with the calculated tolerance factor of $\mathrm{LaBi}_{3} \mathrm{TiNbO}_{9}, \mathrm{SmBi}_{2} \mathrm{TiNbO}_{9}$ and $\mathrm{Bi}_{3} \mathrm{TiNbO}_{9}$ sample (shown as figure 4 inset). To extract more information about compositional-dependent polarization, room temperature Raman studies were performed on the sample.

Figure 5a shows room temperature of Raman spectra of SBTN- $x(x=0.2,0.4,0.6,0.8$ and 1.0) samples, respectively. The overall Raman spectra show broader modes in the two regions $\left(230\right.$ and $\left.260 \mathrm{~cm}^{-1}\right)$. The bands observed near $230 \mathrm{~cm}^{-1}$ represent rocking mode of octahedral structure. The bands observed in the region of $200-400 \mathrm{~cm}^{-1}$ give information about $\mathrm{O}-\mathrm{Ti} / \mathrm{Nb}-\mathrm{O}$ bending [18]. A slight variation observed in figure $5 \mathrm{~b}$ is a clear indication of a structural disorder type, where $\mathrm{Sm}$ ion randomly occupied the Bi-site of the $\left(\mathrm{Bi}_{2} \mathrm{O}_{2}\right)^{2+}$ layer. The modes observed between 300 and $580 \mathrm{~cm}^{-1}$ correspond to a rigid sub-lattice mode, where all the positive and negative ionic displacements may occur with the same magnitude and in the opposite direction. Moreover, the symmetric mode, particularly, observed in the region of $832-837 \mathrm{~cm}^{-1}$ clearly represents symmetric stretching of $(\mathrm{Nb} / \mathrm{Ti}) \mathrm{O}_{6}$ octahedral. The unsymmetrical bands observed in the region of $200-400 \mathrm{~cm}^{-1}$ are also ascribed to either $\mathrm{O}-\mathrm{Ti}-\mathrm{O}$ or O-Nb-O bonding, as mentioned earlier. The inset of figure 5a shows FTIR spectra of SBTN- $x$ samples. A sharp and intensified peak observed near $692 \mathrm{~cm}^{-1}$ for all the samples in FTIR spectra (figure 5a inset) is related to $\left(\mathrm{Bi}_{2} \mathrm{O}_{2}\right)^{2+}$ layers. Another sharp peak appearing at $794 \mathrm{~cm}^{-1}$ is due to Ti-O-Nb bond. The same results can be corroborated with Raman spectra (figure 5). From this, a tentative conclusion is arrived that the two $(\mathrm{Ti} / \mathrm{Nb}) \mathrm{O}_{6}$ octahedra of the present two-layered title compound is connected to the bismuth ion of $\left(\mathrm{B}_{2} \mathrm{O}_{2}\right)^{2+}$ layers, bonded with the edge of oxygen bond of the central octahedra. An important and striking feature of the FTIR data is that all the peak intensities observed for all the compositions at $692 \mathrm{~cm}^{-1}$ are found to be the same. This indicates that the Sm ion occupying Bi site of bismuth oxide layer does not affect the layer structure as it has no effect on the $\mathrm{Bi}-\mathrm{O}$ and $\mathrm{Nb}-\mathrm{O}-\mathrm{Ti}$ interactions.

Raman studies are mainly useful to understand the global effect on dopants and its occupying position [18]. The studies are useful to analyse the ferroelectric polarization of the material. The trivalent Sm easily occupies the A site or Bi-site and satisfies the charge neutrality condition. The A-site or Bi-site vacancies are responsible for the easy rotation of $\mathrm{Nb} / \mathrm{TiO}_{6}$ octahedra in the $a b$-plane compared to the $c$-plane. From the variation of theoretical polarization with the Sm composition (figure 3a), it is observed that the polarization decreases up

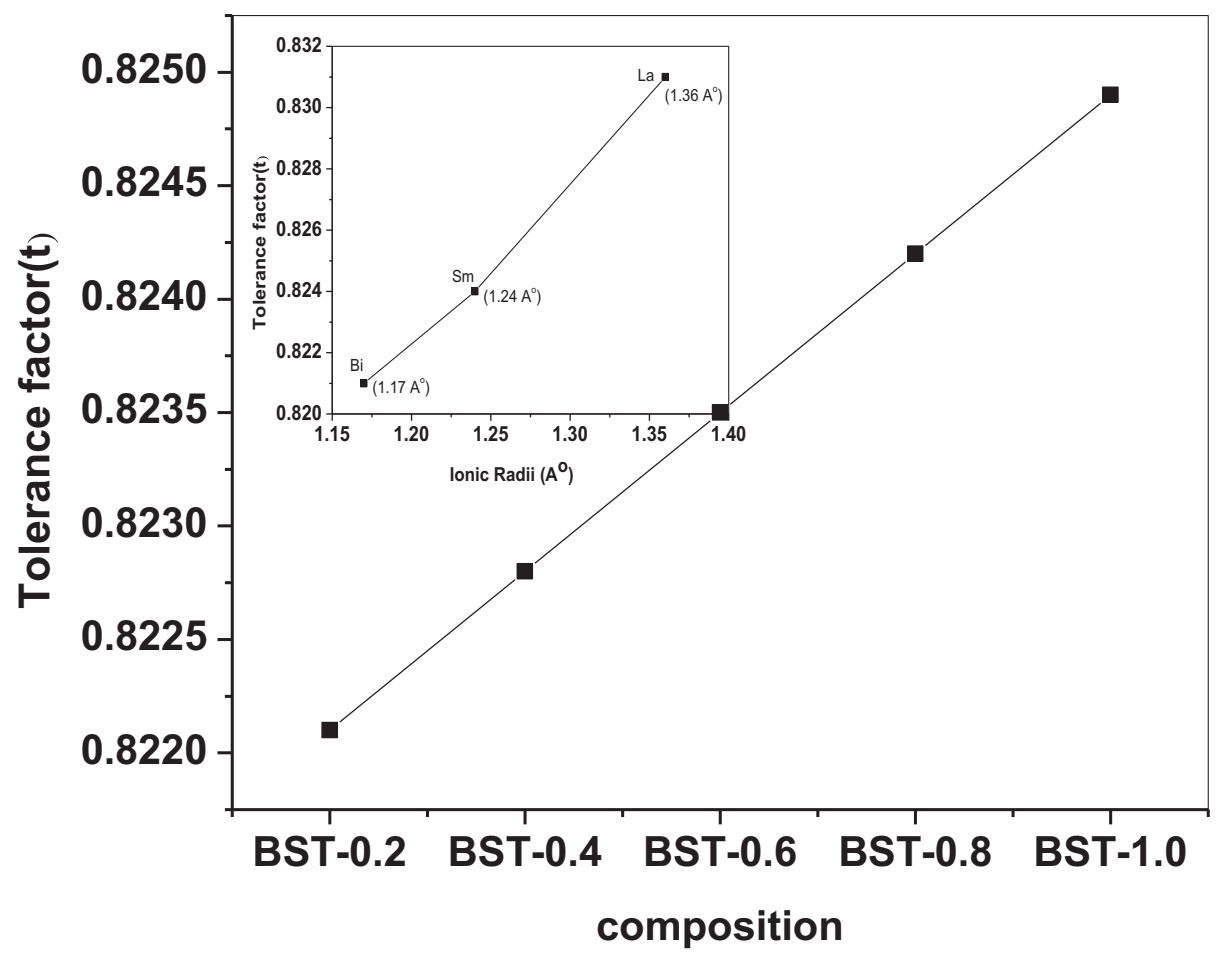

Figure 4. Variation of tolerance factor $(t)$ with the composition SBTN- $x$. Inset: ionic radii $v s$. tolerance factor. 

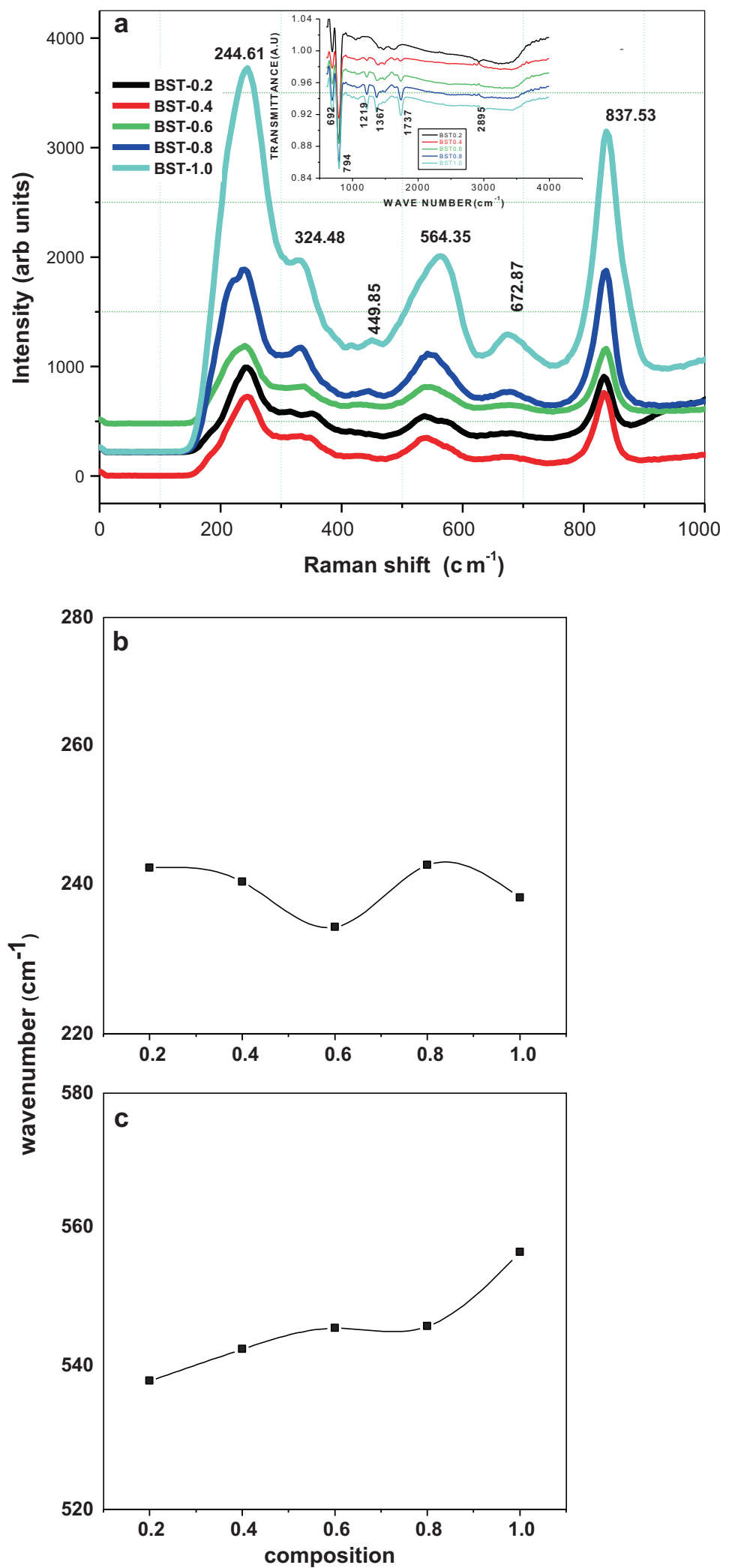

Figure 5. (a) Raman spectra of SBTN- $x$ ceramic at room temperature. Inset: FTIR at room temperature. (b and c) Wave number $\left(\mathrm{cm}^{-1}\right)$ (Raman) vs. Sm composition in the regions of 220-280 and 500-580 $\mathrm{cm}^{-1}$. 


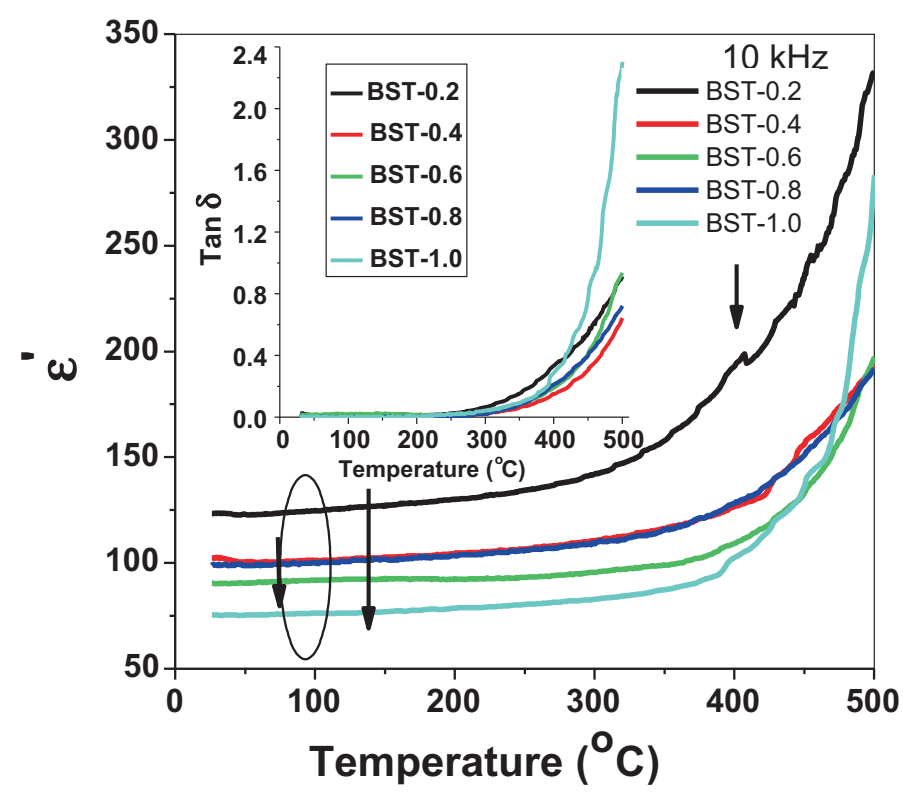

Figure 6. Dielectric constant $v s$. temperature for SBTN-x at $10 \mathrm{kHz}$. Inset: dielectric loss vs. temperature.

to 0.6 composition of $\mathrm{Sm}$ and thereafter, it increases. From the observation, a tentative conclusion is arrived that below 0.6 of $\mathrm{Sm}$ content, the competitive interaction of defects and the octahedra is less predominant and its interaction increases with the composition above 0.6 of Sm content. The results are consistent with the Raman modes observed in the $220-260 \mathrm{~cm}^{-1}$ region. Moreover, the total polarization, theoretically calculated from the different constituent ions is found be more when compared to the experimentally calculated remnant polarization. The reason is ascribed to the defects, which play a role in the rotation of octahedral [18-26].

Figure 6 shows the variation of dielectric constant with temperature, obtained at $10 \mathrm{kHz}$, for all the compositions. The inset of figure 6 shows the variation of dielectric loss with temperature for all the samples, respectively. From the plot, it is observed that the dielectric constant increases dramatically above $400^{\circ} \mathrm{C}$. However, acceptor-doped BTNO $\left(\mathrm{Bi}_{3} \mathrm{Nb}_{0.8} \mathrm{Ti}_{1.2} \mathrm{O}_{9}\right)$ samples showed the dielectric loss peaks near $300^{\circ} \mathrm{C}$ [9]. It clearly shows that the amount of dopant or type of dopant, certainly affects the transition temperature. Newnham et al [8] reported that BTNO belongs to weak-firstorder type and therefore, the transition movement was found to be gradual-continuous movement of domain state, unlike first-order transitions. The possible reason for not observing any dielectric peak below $400^{\circ} \mathrm{C}$ in the present investigation is attributed to the presence of $\mathrm{Nb}$ ion, which might have created complex defect dipoles.

As mentioned earlier, the general formula of the present layered structure is $\left(\mathrm{Bi}_{2} \mathrm{O}_{2}\right)^{2+}\left(\mathrm{A}_{n-1} \mathrm{~B}_{n} \mathrm{O}_{3 n+1}\right)^{2}$. Consider $\mathrm{A}$-site and $\mathrm{B}$-site charges as $\mathrm{A}^{x+}$ and $\mathrm{B}^{y+}$, then the general charge balancing equation can be written as: $x(n-1)+y(n)$
$-2(3 n+1)=-2$ or $(x n-x+y n+6 n-2=-2)$. The equivalent equation for the above is: $n(x+y-6)=x$. Since the value of $n$ (no. of layers) is 2, the possible value of $x$ and $y$ is 2 and 5 . Based on the $\mathrm{ABO}_{3}$ defect formula, one can conclude that the A- and B-sites are occupied by di and pentavalent ions only. However, in the present case, the A-site is occupied by trivalent ion and the B-site is occupied by both tetra and pentavalent ions. The ionic radii of A-site ions were found to be in the order of $\mathrm{La}^{3+}>\mathrm{Sm}^{3+}>\mathrm{Bi}^{3}$. From this, one can conclude that the rattling space of $\mathrm{Sm}$-modified $\mathrm{Bi}$ is more than the La-modified Bi ion. The results are seen in figure 4.

The parent compound $\left(\mathrm{Bi}_{3} \mathrm{TiNbO}_{9}\right)$ and the present title compounds can be represented with the following $\mathrm{ABO}_{3}$ based molecular formula:

$$
\begin{aligned}
& \mathrm{Bi}_{3} \mathrm{TiNbO}_{9}=\mathrm{Bi}_{2} \mathrm{O}_{3} \cdot 2\left(\mathrm{Bi}_{1 / 2}[]_{1 / 2} \mathrm{Nb}_{1 / 2} \mathrm{Ti}_{1 / 2} \mathrm{O}_{3}\right), \\
& \mathrm{Sm}_{x} \mathrm{Bi}_{3-x} \mathrm{TiNbO}_{9} \\
& \quad=\mathrm{Bi}_{2} \mathrm{O}_{3} \cdot 2\left(\mathrm{Sm}_{x / 2} \mathrm{Bi}_{(1-x) / 2}[]_{1 / 2} \mathrm{Ti}_{1 / 2} \mathrm{Nb}_{1 / 2} \mathrm{O}_{3}\right) .
\end{aligned}
$$

Here, the bracket [ ] term mentioned in equation (2) represents the unoccupied states. These unoccupied sites certainly trap the electron and form defect dipoles. The defect dipoles are called complex defect dipoles which are generally responsible for not observing a broad dielectric peak or relaxor like behaviour near $400^{\circ} \mathrm{C}$. If $\mathrm{La}$ ion is substituted in place of $\mathrm{Bi}$, on account of its high ionic radius, La ion gets diffused and therefore, the unoccupied site and electron forms a vacancyelectron dipole. In addition, rattle space for B-site ion may increase and in turn reflects into broad dielectric or diffusedlike behaviour for LBTN. On the other hand, the tendency of 
Sm diffusing into the Bi-site is less and might decrease the rattle space for $\mathrm{B}$-site.

The variation of modulus (complimentary to dielectrics) with the frequency shows the dielectric relaxation processes present in the samples. In the present investigation, modulus data was extracted from the impedance data, using the following relation:

$$
\begin{aligned}
M^{*}(\omega) & =M^{\prime}+i M^{\prime \prime}=j \omega C_{\mathrm{o}} Z^{*}(\omega), \\
& =M_{\infty}\left[\int_{0}^{\infty}\left(-\frac{\mathrm{d} \phi}{\mathrm{d} t}\right) \exp (i \omega t) \mathrm{d} t\right],
\end{aligned}
$$

where $M_{\infty}\left(=\varepsilon_{\infty}\right)^{-1}$ is the extrapolation value of $M^{\prime}$ in the frequency explicit plot. The term $\phi$ and $C_{\mathrm{o}}$ representing time evolution of applied field and geometrical capacitance of the sample, respectively. Figure $7 \mathrm{a}$ and $\mathrm{b}$ shows the variation of real part and imaginary part of modulus as a function of frequency obtained at $450^{\circ} \mathrm{C}$ for all the samples. Both $M^{\prime}$ as well as $M^{\prime \prime}$ values are found to increase with increasing temperature and the $M^{\prime \prime}$ maximum peak is found to shift towards higher frequency side with increasing temperature (figures are not presented). The overall asymmetric nature of $M^{\prime}$ plots indicates the stretched exponential nature with different relaxation time values. The broad relaxations observed in the $M^{\prime \prime}$ plot (figure 7b) are attributed to the presence of grain and grain boundary behaviour of the sample. From the inset of figure $7 \mathrm{a}$, it is clear that the inter-layer distance decreases with increasing $\mathrm{Sm}$ composition. The full width at half maxima (FWHM) obtained for [ [ $\left.\begin{array}{lll}1 & 1 & 5\end{array}\right]$ reflection is found to increase with increasing Sm composition. The position of the maximum peak obtained in the spectroscopic data is found to shift towards a higher frequency side with increase in the temperature. This typical thermally activated Debye relaxation process is once again considered to be the characteristic
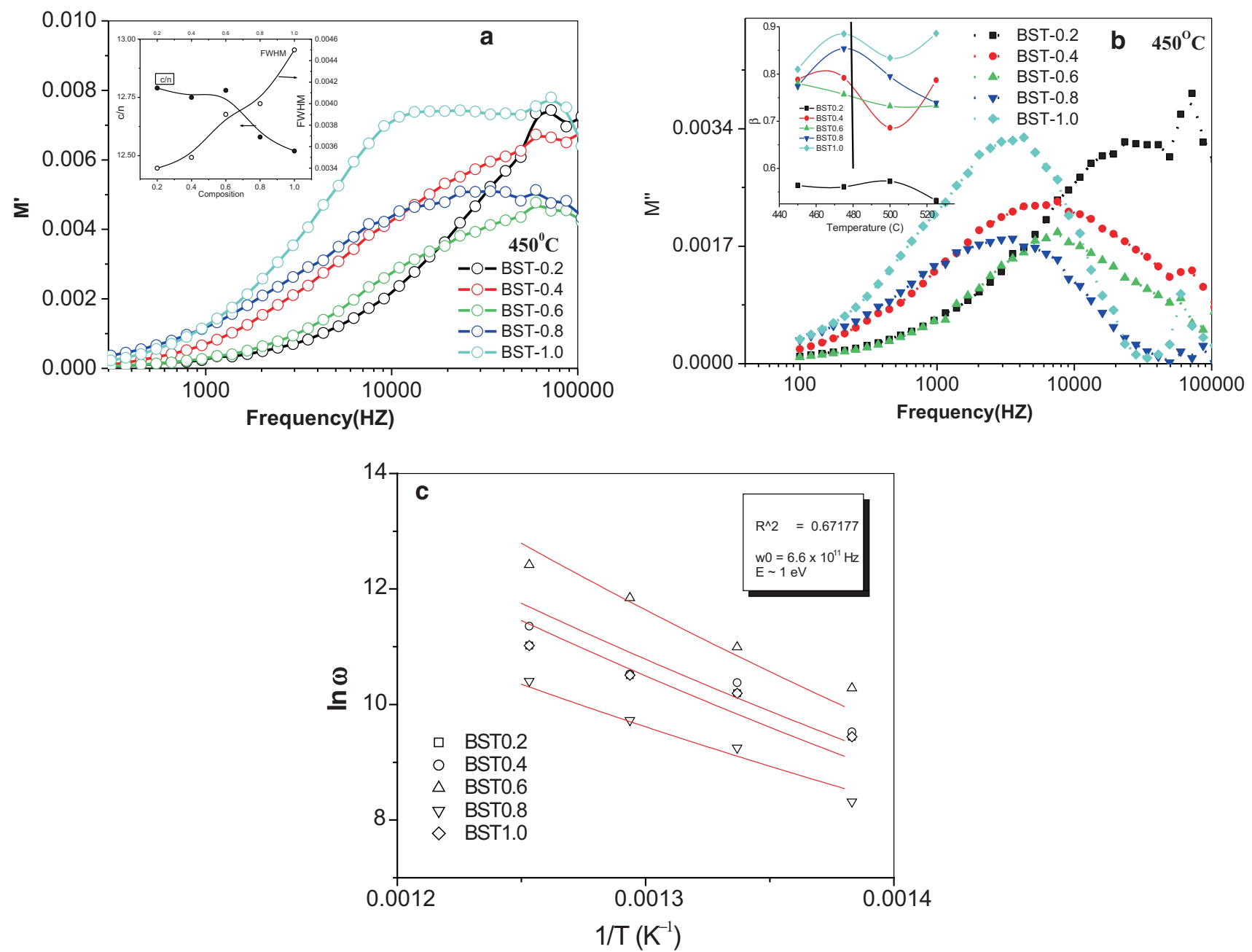

Figure 7. (a) Variation of real part of modulus with frequency obtained at $450^{\circ} \mathrm{C}$ for all compounds. Inset: Sm composition $v s$. interlayer distance $(c / n)$ and FWHM obtained for maximum XRD intensity peak [ 11 5]. (b) Variation of imaginary part of modulus with frequency obtained at $450^{\circ} \mathrm{C}$ for all the different compositions. Inset: the stretched exponential parameter $v s$. temperature for all the samples. (c) $\ln \omega(=2 \pi f$, where $f$ is maximum spectroscopic frequency) $v s$. inverse of temperature for SBTN- $x$. 

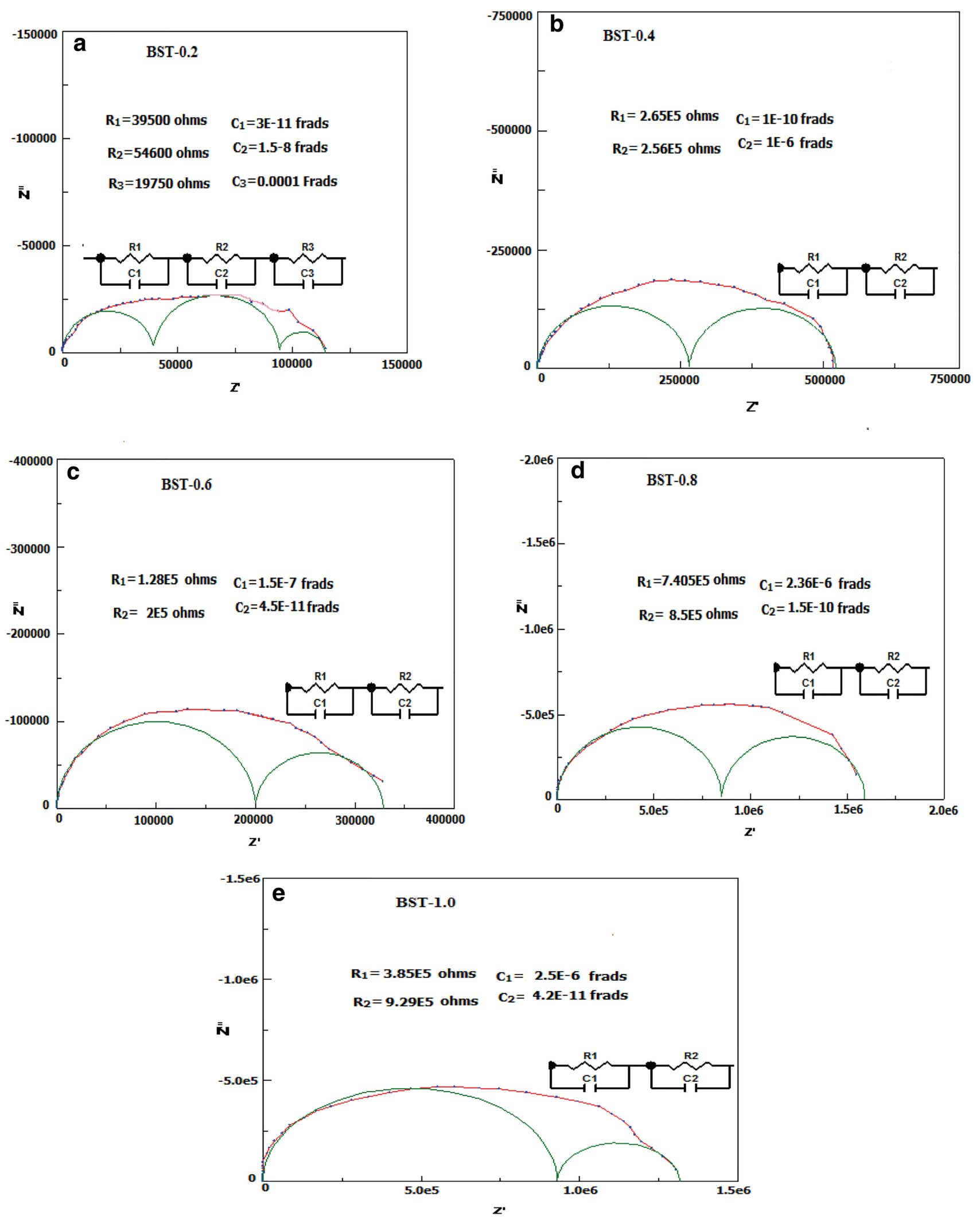

Figure 8. (a-e) Complex impedance plots (Cole-Cole plots) of SBTN- $x$. 
feature of these compounds. The logarithmic peak frequency is plotted against inverse temperature (figure 7c) and showed a straight line. The data are found to be well-fit with the following Arrhenius equation:

$$
\omega=\omega_{0} \exp (-E / k T)
$$

where $E$ is the activation energy and $k$ and $T$ have their usual significance. The activation energy for all these compounds are found to be roughly $1 \mathrm{eV}$ and the characteristic frequency is in the range of $10^{11} \mathrm{~Hz}$. These values are good in agreement with the Shulman et al [19] results. Based on the activation energy values $(1 \mathrm{eV})$, one can say that the relaxations are mainly due to the oxygen ion jump mechanism.

The real and imaginary parts of the impedance measured at $450^{\circ} \mathrm{C}$ is shown in figure $8 \mathrm{a}-\mathrm{e}$ for all the samples under the study (Cole-Cole plots). The impedance data was found to be well-fit into two semicircles, and the equivalent circuit model is also depicted in figure 8 . The centres of the resolved semicircle are not lying on $x$-axis, instead they are depressed with an angle $\theta$. The depressed semicircular nature as well as asymmetric and broad nature of $M^{\prime \prime}$ plots (called non-Debye nature) indicate the non-exponential (Kohlrausch) relaxations. This nature suggests that ionic migration is trough hopping type. Here, it should be noted that the stretching exponential is a measure of degree of interaction between charge carriers and the stretched parameter which is more or less related to the depressed angle $\theta$ by the relation: $\theta=(1-\beta) \pi / 2$. The variation of $\beta$-parameter or stretched exponential parameter with temperature is shown in the inset of figure $7 \mathrm{~b}$. A change in $\beta$-parameter for all the compositions, shown as solid line in the inset of figure $7 \mathrm{~b}$ indicates the phase transition temperature. Here, it is not ruled out that the dynamic dielectric phenomenon may be different from the apparent dielectric phenomenon. It is also evident from the inset of figure $7 \mathrm{~b}$ that $\beta$ value is $>0.5$ throughout the investigation. It is a clear indication where charge carrier becomes more mobile at this temperature. The reason for not observing a prominent hump below $300^{\circ} \mathrm{C}$ is attributed to the oxygen ion loss by forming a complex defect dipole. Since the moment of these defect dipoles is difficult, one cannot observe any humps in dielectric as well as saturation in hysteresis loops. The results were consistent with our earlier results [13-16,25]. The observation of a small hump in dielectric data (shown as arrow in figure 4) above $400^{\circ} \mathrm{C}$ is attributed to the rotation of oxygen octahedra. The decrease in dielectric constant with increase in the $\mathrm{Sm}$ composition (shown as encircled in figure 6) is attributed to the increase in tolerance factor (figure 3), where the tolerance factor gives information about the rattling space available at B-site. The increase in the size of the A-site cation certainly affects the rattling space of the B-site ion. The results were indeed consistent with the dielectric data of $\mathrm{Bi}_{2.45} \mathrm{La}_{0.55} \mathrm{TiNbO}_{9}$, where a broad dielectric peak was observed [14].
A small dielectric hump observed at $400^{\circ} \mathrm{C}$ is attributed to the entrapment of electron with the oxygen vacancy. The oxygen complex clusters generate localized states in the band gap and inhomogeneous charge distribution in the cell thus, allows for electron trapping. The localized levels are distributed energetically, so that various energies are able to excite the trapped electrons. The following Kroger-Vink model suggests that increase in the temperature reduces the disorder and creates electron-captured oxygen vacancies, according to equations:

$$
\begin{aligned}
& {\left[\mathrm{NbO}_{5} \cdot \mathrm{V}_{\mathrm{O}}^{x}\right]+\left[\mathrm{NbO}_{6}\right]^{x} \rightarrow\left[\mathrm{NbO}_{5} \cdot \mathrm{V}_{\mathrm{O}}^{\prime}\right]+\left[\mathrm{NbO}_{6}\right]^{\prime},} \\
& {\left[\mathrm{NbO}_{5} \cdot \mathrm{V}_{\mathrm{O}}^{\prime}\right]+\left[\mathrm{NbO}_{6}\right]^{x} \rightarrow\left[\mathrm{NbO}_{5} \cdot \mathrm{V}_{\mathrm{O}}^{\prime \prime}\right]+\left[\mathrm{NbO}_{6}\right]^{\prime},} \\
& {\left[\mathrm{NbO}_{5} \cdot \mathrm{V}_{\mathrm{O}}^{\prime \prime}\right]+\frac{1}{2} \mathrm{O}_{2} \rightarrow\left[\mathrm{NbO}_{6}\right],}
\end{aligned}
$$

where $\left[\mathrm{NbO}_{6}\right]^{\prime}$ is donor, $\left[\mathrm{NbO}_{5} \cdot \mathrm{V}_{\mathrm{O}}^{\prime}\right]$ is donor-acceptor and $\left[\mathrm{NbO}_{5} \cdot \mathrm{V}_{\mathrm{O}}^{\prime \prime}\right]$ is acceptor. Here, the oxygen vacancies from complexes defect dipoles in the intermediate structure. In this structure, $\left[\mathrm{NbO}_{5} \cdot \mathrm{V}_{\mathrm{O}}^{x}\right]$ complex cluster is a donor candidate and $\mathrm{NbO}_{6}^{x}$ is an acceptor candidate. We speculated that these oxygen complex vacancies induce new energy in the band gap and can be attributed to the niobium-oxygen complex vacancy centres. This is evident from the stretched exponential parameter with temperature plot.

\section{Conclusions}

The modes observed in the Raman spectra near $240 \mathrm{~cm}^{-1}$ seems to be unchanged for different Sm modifications for the $\mathrm{Bi}$ site, clearly indicating that the $\mathrm{Sm}$ ion readily substitutes $\mathrm{Bi}$ ion of $\mathrm{Bi}_{2} \mathrm{O}_{2}$ layers, and certainly not perovskite blocks. The results are consistent with the tolerance factor values. No splitting observed near $200 \mathrm{~cm}^{-1}$ clearly confirms that the Sm ion is linked with $\left(\mathrm{Bi}_{2} \mathrm{O}_{2}\right)^{2+}$ layers, but certainly not with perovskite blocks. The sharp peaks appearing in FTIR spectra at $692 \mathrm{~cm}^{-1}$ can be corroborated to the same (Bi-O vibrations). Generally, in most of the BLSF materials, a hump is observed in the dielectric measurements near $300^{\circ} \mathrm{C}$. However, in the present case, we could not observe any peak in the dielectric measurements. This is an indication of electron trapped with the oxygen vacancy. The oxygen complex clusters generate localized states in the band gap and inhomogeneous charge distribution in the cell thus, allows for electron trapping. The localized levels are distributed energetically, so that various energies are able to excite the trapped electrons.

\section{Acknowledgements}

One of the authors (MAB) thanks the management of Deccan College of Engineering and Technology for constant encouragement. We are thankful to the referee for the useful discussions. 


\section{References}

[1] Paz de Araujo C A, Cuchiaro J D, McMillan L D, Scott M C and Scott J F 1995 Nat. Lond. 374627

[2] Park B H, Kang B S, Bu S D, Noh T W, Jo J W and Lee J 1999 Nat. Lond. 401682

[3] Scott J F and Paz de Araujo C A 1989 Science 246 1400

[4] Cao G Z 2001 Advances in materials science and applications (ed) D L Shi (Beijing: TUP and Springer) p 86

[5] Subba Rao E C 1961 Phys. Rev. 122804

[6] Subba Rao E C 1962 J. Phys. Chem. Solids 23665

[7] Aurivillius B 1949 ArkiKemi 1463

[8] Newnham R E, Wolfe R F, Horsey R S, Diaz-Colon F A and Kay M I 1973 Mater. Res. Bull. 81183

[9] Yan H, Zhang H, Zhang Z, Ubic R and Reece M J 2006 J. Eur. Ceram. Soc. 262785

[10] Zhang Z, Yan H X, Dong X L and Wang Y L 2003 Mater. Res. Bull. 38241

[11] Zhou Z, Dong X and Huang S 2006 J. Am. Ceram. Soc. 89 2939

[12] Wu Y, Forbess M J, Seraji S, Limmer S J, Chou T P, Nguyan C et al 2001 J. Appl. Phys. 905296

[13] Dubey S and Kurchania R 2015 Bull. Mater. Sci. 38 1881
[14] Prasad N V, Puli V S, Pradhan D K, Gupta S M, Prasad G and Katiyar R S 2015 J. Ferroelectr. 47429

[15] Sugaya Y, Shoji K and Sakata K 2003 Jpn. J. Appl. Phys. 42 6086

[16] Abdul Basheer M D, Prasad G, Kumar G S and Prasad N V 2017 J. Ferroelectr. 51775

[17] Thirumal Reddy N, Prasad N V, Kumar G S, Prasad G and Ramana E V 2013 J. Ferroelectr. 447126

[18] Qian Y, Wang X, Zhu J, Mao X and Chen X 2007 J. Rare Earths 25240

[19] Shulman H, Damjanovic D and Setter N 2000 J. Am. Ceram. Soc. 83528

[20] Wang Z, Chen T, Zhu W, Fu J, Yan H and Li C 1998 Acta Phys. Sin. OVED 7764

[21] Coondoo I, Agarwal S K and Jha A K 2009 Mater. Res. Bull. 44 1288

[22] Sridarane R, Subramanian S, Janani N and Murugan R 2010 J. Alloys Compd. 492642

[23] Nayak P, Mohapatra S R, Kumar P and Panigrahi S 2015 Ceram. Int. 419361

[24] Pribosic I, Makovec D and Drofenik M 2001 J. Eur. Ceram. Soc. 211327

[25] Prasad N V, Prasad G, Kumar G S and Gupta S M 2012 Ferroelectrics $\mathbf{4 3 7} 88$

[26] Liu G Z, Wang C, Gu H S and Lu H B 2007 J. Phys. D: Appl. Phys. $\mathbf{4 0} 7817$ 